\title{
A Pervasive Telemedicine System Exploiting the DVB-T Technology
}

\author{
Gianmarco Angius, Danilo Pani, Luigi Raffo, Stefano Seruis \\ Dept. of Electrical and Electronic Engineering \\ University of Cagliari \\ 09123 Cagliari, Italy \\ Email: \{g.angius,pani,luigi\}@diee.unica.it
}

\author{
Paolo Randaccio \\ Dept. of Physics \\ University of Cagliari \\ 09042 Monserrato (CA), Italy \\ Email: paolo.randaccio@ca.infn.it
}

\begin{abstract}
Telemedicine is a form of remote assistance to patients unable to easily reach the hospitals. The diffusion of such systems depends both on the availability of widespread low-cost platforms and on the ease of use. In this paper, a DVB-T based platform for telemedicine is presented. The proposed system enables to perform simple routine exams through a low-cost Base Station connected to the patient's DVB-T set-top box, using the remote control and the TV screen as I/O interfaces for the user. The uplink connection is used to send the results to a remote care center. The practice with such home-entertainment equipments allows even elderly people to easily exploit the proposed platform.
\end{abstract}

\section{INTRODUCTION}

Telemedicine is an interesting example of pervasive healthcare system, aimed to enable patients with chronic diseases to be assisted remotely by the care staff without the need for frequent visits to the hospital. Beyond the advantages for the patients, the costs for the public administration can be reduced exploiting low-cost equipments to create the infrastructure. Until now, the largest part of such systems was based on a PC with an internet connection (e.g. [1]) or on expensive dedicated devices (e.g. [2], [3], [4]). Often, they are not intuitive for untrained people (or not conceived with this aim [5]) and then their use is intended only for young people, more accustomed to digital equipments but at the same time not representing the main telemedicine target. Furthermore, if a PC is not present in the patient's home, the cost to set-up the platform could be high.

After 2012, Digital Video Broadcast Terrestrial (DVB-T) will be the only terrestrial television system. Any TV set can be used with DVB-T providing that an external set-top box is properly connected. Taking into account the wide diffusion of TV sets and the very low cost of DVB-T set-top boxes, the development of a telemedicine system based on such technology could represent the answer to the problems raised by the other solutions.

In this paper, a prototypal device and the whole system framework for a DVB-T based telemedicine system are presented. In such a system, the patient is able to interact with the health-monitor device through the TV screen and the remote controller of the set-top box. The application running on the set-top box is provided by a Digital Video Broadcaster, so that the software maintenance and upgrade can

Permission to make digital or hard copies of all or part of this work for personal or classroom

use is granted without fee provided that copies are not made or distributed for profit or commercial

advantage and that copies bear this notice and the full citation on the first page. To copy otherwise,

to republish, to post on servers or to redistribute to lists, requires prior specific permission and/or a fee.

PERVASIVEHEALTH 2008, 30 Jan - 1 Feb. Tampere, Finland

Copyright $\odot 2008$ ICST 978-963-9799-15-8

DOI 10.4108/ICST.PERVASIVEHEALTH2008.2507 be easily accomplished without any direct user intervention. The adoption of the interactive set-top box, which embeds a modem for a data uplink connection, allows the transmission to a Remote Care Center of the examination results over the telephone network without a personal computer or any dedicated device. A personal smart card enables the patient to configure the application for his/her needs without any direct intervention. Beyond the standard home entertainment equipments, the system uses a low-cost prototypal Base Station to perform the acquisition of the patient's signals. This unit is completely controlled by the application running on the set-top box that provides also the interface for the patient. To validate the system we developed a proof-of-concept Base Station limited to the acquisition of a 1-lead electrocardiogram (ECG).

The remainder of this paper is organized as follows. In Section II the whole system is introduced; in Section III the application on the set-top-box with the user interface is presented, whereas some details on the hardware Base Station are given in Section IV. In Section V a short description of the application broadcasting technique is given. Section VI concludes this work.

\section{System Overview}

The system presented in this paper was conceived to keep as low as possible the costs of the extra-hardware with respect to a minimum setup composed of a TV and a DVB-T set-top box connected to the telephone line. This way the usability by untrained people not accustomed to digital equipments but the TV set one is also preserved. The system is depicted in Figure 1. It is composed of two main parts hosted respectively in the Remote Care Center (RCC) and at the patient's home.

On the RCC there is only a simple PC acting as TCP/IP server. The server receives the data stream and saves some files on a database directory: a patient identification file, providing all the information needed to identify the patient, and some exam data files. For exams such as ECGs, requiring a graphical visualization on the RCC, such data files are accompanied by a header file specifying the information useful for the correct visualization (e.g. sample rate). The same does not hold for exams such as arterial blood pressure, temperature, etc. 


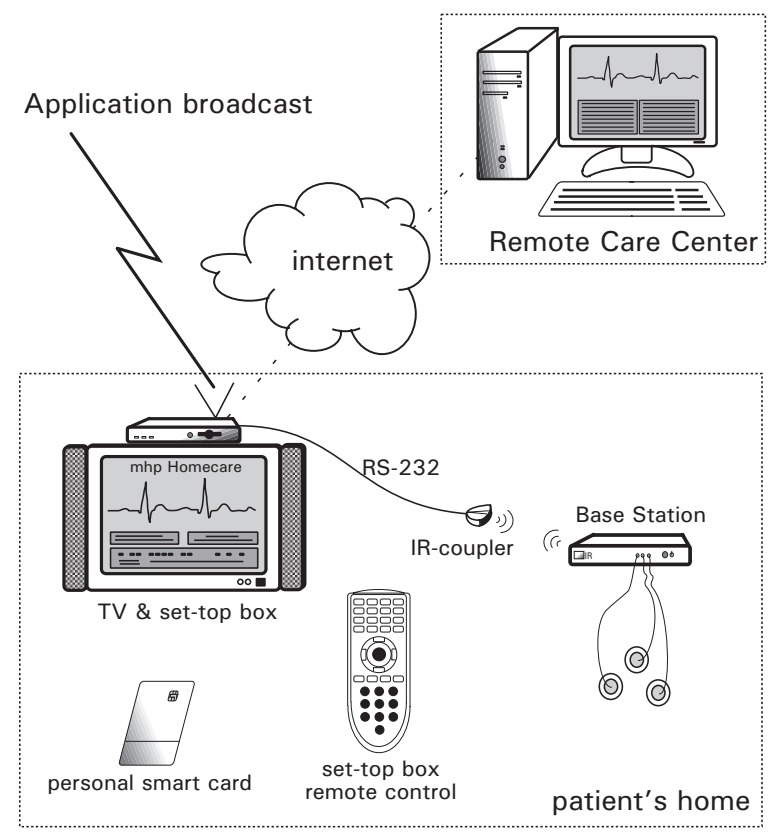

Fig. 1. The main parts of the proposed telemedicine system.

On the patient's side, beyond the TV set, there is only a local microcontroller-based Base Station (BS) connected to the set-top box through its RS-232 serial port. The BS directly provides some examination features (in this prototypal version only a 1-lead ECG), and can be expanded to embed both other examination hardware and interfaces to connect some commercial devices (such as glucose meters, coagulometers, etc.). It is connected to the set-top box for data visualization and transmission, and it is controlled by it and then indirectly by the user only through the remote control. A personal smartcard (the largest part of DVB-T set-top box provides the smartcard slot for pay-per-view services) has a twofold role: identification and configuration, as we will see in Section III-A.

For this work, we used the Telesystem TS7.4DT set-top box, with the version $21 \mathrm{p} 1$ of the producer's software, and implementing the MHP 1.02 profile with some enhancements, primarily the addition of java packages for both the smart card and the serial RS-232 port management. The real-time OS is Osmosys. The MHP set-top box acts as a (slow) PC, having user I/O devices (remote control and TV screen), communication ports (RS-232) and an internal modem beyond the traditional audio/video connectors.

\section{THE SOFTWARE: MHPHOMECARE}

Since the proposed telemedicine system is based on a DVB$\mathrm{T}$ platform, it is worth to analyze at first our application running on the patient set-top box, MHPHomecare, which manages the whole system. It is an Xlet, i.e. a JavaTV application model following a well defined lifecycle [6]. The Xlet is loaded into the set-top box by means of a broadcast transmission provided by a broadcaster (Section V). Once MHPHomecare has been loaded, if the patient activates it by means of the remote control as for any other MHP application,

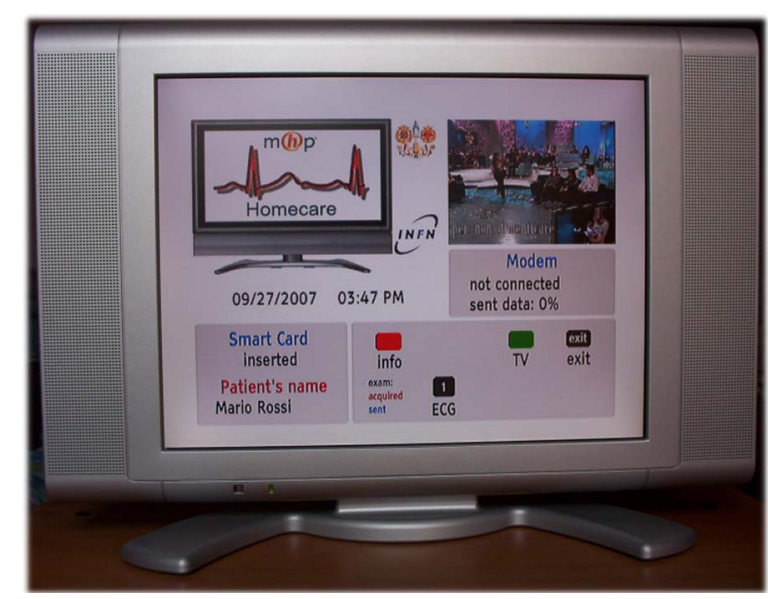

Fig. 2. A shot of the main screen of the MHPHomecare application (primary FSF) on an LCD TV.

the graphic interface is launched providing interactivity with the patient.

\section{A. MHPHomecare Patient Interface}

The patient interacts with the application providing control commands through the remote control and analyzing their effects through the TV screen, where the different Full-Screen Frames (FSF) of the application are shown.

At first, a primary FSF is shown (Fig. 2), waiting for the insertion of a valid identification smart card into the appropriate set-top box slot. Without any valid identification card the application cannot proceed. The smart card is read by means of the SATSA (Security and Trust Services API) classes [7], that represent the new standard for smart card access in Java. For this research work we used the ACOS2 microprocessor card by Advanced Card Systems Ltd. The application reads from the card the User Data File, which stores the patient's name and its personal identification code, the treating physician's name and its identification number, a permission exam code, some information about the ISP (user ID, password, telephone number) and the RCC server IP address. This way the patient does not have to remember any code, number, password, etc. The RCC must program the smart card for a patient personalizing such information, so that different patients can share the same hardware platform simply inserting their personal smart card. For instance, the permission exam code is used to allow the patient to perform only some specific exams among those available, hence disabling all the other ones. The smart-card must still in place until all the examination procedures are concluded. A small area on the top-right corner of this FSF is devoted to the background TV program streaming (Fig. 2). From the primary FSF, the patient can chose the exam to perform (a new FSF for that exam will be loaded), send the results of all the performed exams to the RCC (if any), review the performed exams. A pop-up window alerts the patient if he/she is exiting the primary FSF without sending the results to the RCC (the exam results will be lost).

At the moment the application supports only one exam, i.e. 


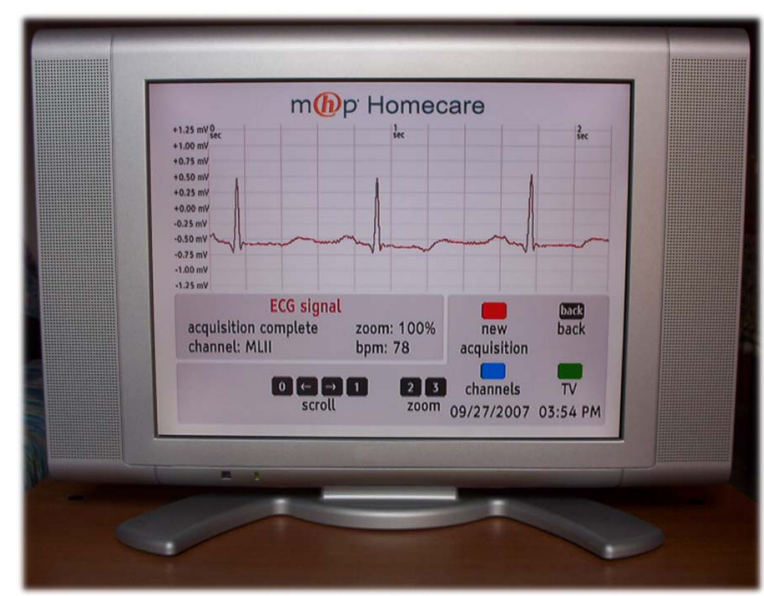

Fig. 3. A shot of the ECG exam screen of the MHPHomecare application (ECG Exam FSF) on an LCD TV.

a single lead ECG or a 3-lead ECG. A screenshot of this FSF for a 1-lead ECG is depicted in Fig. 3. Hereafter we'll describe the ECG exam FSF in the more complex case (3-lead). This FSF enables the ECG acquisition and shows in real-time the samples acquired from the BS and all the other information sent by it about the current exam, such as the hearth rate and warning messages on signal quality. Before starting the signal acquisition, the user can choose to see in real time a single lead or all the acquired lead. As in any commercial ECG device, the actually sampled main leads are only 2 ( $I$ and $I I)$ whereas the other one $(I I I)$ is computed as $I I I=I I-I$. Once the exam has been saved, from the same FSF it is possible to review it on the TV, with a scroll feature, and to zoom on a single lead. The patient can also redo the same exam: a pop-up alerts the patient that the previous record will be overwritten. Pressing the Back key on the remote control, the application returns to the primary FSF with the possibility to send the record to the RCC via the internal modem, simply pressing a key. Every FSF can be reduced to allow the patient to watch a TV program while the application is running in background.

\section{B. Set-top box to BS interface}

The control of the BS from MHPHomecare takes place by means of an RS-232 serial port connection. To avoid direct coupling with the $\mathrm{AC}$ mains, the serial connection is IR-coupled. The communication is managed through the it.dtt.comm Java package and the serial port behaves according with the RS-232 standard. The set-top box sends to the BS the commands to start the acquisition and to stop it. The "start" command is logically divided in two parts, i.e. the actual command to enable the serial port transmission, and a code that identifies the exam the patient chose by means of MHPHomecare. The "stop" command word is undifferentiated. The Xlet deals with 8 or 16-bit signals (the data are stored in arrays of short). In the current implementation only the ECG is available on the BS, and according to its sample rate and data width (Section IV), the maximum painting rate of the graphic area where the signal is plotted is $200 \mathrm{~ms}$. With this timing, even if the set-top box receives the signal samples from the BS sample by sample, the Xlet uses a buffer of $50 \times 2$ samples at a time for computation and display purposes. MHPHomecare stops the signal acquisition if:

- the user presses the appropriate key on its remote control;

- the signal quality is too poor (e.g.: misplaced electrodes), displaying a message on the exam FSF;

- the default exam time (10 seconds for the ECG) has been reached.

After the exam stops with a "stop" command word, the BS sends to the set-top box a last word with the value of the heart rate expressed in bpm so that the proper FSF can be updated.

\section{HARdware: the Base Station}

In order to test our system we developed a simple BS with a serial interface. According to the international recommendations about the patent's safety, the physical connection between the BS and the set-top box is represented by an infrared link. The BS embeds a module for the IR transmission of the serial stream, whereas the set-top box exploits an external IR coupler connected to the RS-232 serial port. From the patient's point of view, the BS is a black box without any own user interface: it is controlled by the patient through the remote control of the set-top box. The BS is battery powered, for simpler use and improved patient safety. At this moment, it implements a single-lead ECG (lead I) and consists in a classical ECG amplifier coupled with a very simple and low cost Digital Signal Controller (DSC), i.e. the Microchip ${ }^{T M}$ dsPIC30F4013. This is a 16-bit 30MIPS DSC enhanced with DSP hardware, such as a 17-bit x 17-bit multiplier, a 40bit ALU, two 40-bit saturating accumulators and a 40-bit bidirectional barrel shifter. It also provides several embedded peripherals such as a 12 bit successive approximation analog to digital converter, and an UART, both of them employed in this prototype.

The ECG amplifier is a low-pass system with a variable gain up to 2000 and a cutoff frequency of $100 \mathrm{~Hz}$. It consists of an optically isolated instrumentation amplifier with high CMRR connected to the wrists of the patient and an inverting operational amplifier connected to the right ankle for electromagnetic coupling noise rejection purpose, so that only 3 disposable electrodes can be used. The DSC is programmed to sample the ECG signal at $250 \mathrm{~Hz}$. The sampled signal is digitally filtered to remove the mains interference and the baseline drift. We also implemented a simple QRS detector for heart rate calculation and visualization on the TV screen. The heart rate is calculated on the overall signal but the QRS detector works on-line sample by sample. The QRS detector is based on both the signal and its first derivative amplitudes, and is a modified version of the algorithm presented in [8]. It was chosen for its simplicity, since the indication to be provided is only for a qualitative analysis (it can be performed again on the RCC). The RR interval measurement is performed sample by sample, and if it is too far from the standards a warning code is sent to the set-top box to ask the user to control the correct connection of the electrodes. The average heart rate is 
sent when the acquisition stops and is referred to the average RR during the acquisition window. All these computations are performed in real-time on the BS, since the set-top box is quite slow in processing due to the Java VM and to the applications running on it. The 16-bit samples are serialized for the communication with the set-top box, the baud-rate being 19,200 .

\section{BROADCASTING MHPHOMECARE}

The application is loaded on the patient's set-top box through an ether broadcast DVB-T transmission, while the user is watching the broadcaster's channels. In general, a DVB-T broadcast system is designed to transmit a compressed digital audio/video/data MPEG-2 stream, using Orthogonal Frequency Divisional Multiplexing and a modulation technique compatible with the traditional $8 \mathrm{MHz}$ bandwidth of the analog transmission channel (i.e. QPSK, 16QAM and 64QAM). The compressed video, audio, and data streams of one TV channel are multiplexed together to form a Programme Elementary Stream (PES). The basic digital stream that a settop box can receive is an MPEG-2 Transport Stream (TS), which is formed joining together one or more PES. From the receiver's point of view, all the TV channel and services are received at once but it only demultiplexes and then decodes the selected content, one at a time, from the received TS.

Our DVB-T broadcast system is depicted in Fig. 4 and it is based on the CreaTV MiniLab by Media Solution. Through the JustDvb-It 2.0 software [9], video and audio MPEG-2 encoded contents are multiplexed together with the Xlet application to build the Single Program Transport Stream (SPTS) to broadcast. A PCI card DekTec DTA-110, a multistandard modulator with UHF Upconverter, is coupled with a $20 \mathrm{dBm}$ RF amplifier (RFbay LPA-4-14) for the ether transmission of the SPTS. To establish the bitrate needed to encode audio, video and multimedia contents in the MPEG-2 format, it is necessary to know the coding and modulation parameters used to transmit the SPTS. We have chosen $1 / 2$ for the convolutional rate, 16QAM for the modulation type and $1 / 4$ of the original block length for the guard interval. The resulting bitrate is 9953 Kbps.

\section{CONCLUSIONS}

In this paper we presented a DVB-T based telemedicine system. The widespread diffusion of the TV sets and the relatively low cost of the DVB-T set-top boxes create the perfect scenario for the introduction of such a technology for the remote non-continuous monitoring of young and elderly people. Compared to traditional dedicated systems, the presence of a visual environment on the TV screen allows a more friendly approach providing also detailed information and feedbacks about the signal quality, and guiding the user through all the exam procedure without any required printed manual. We successfully implemented a broadcasting system to load the Xlet application on the set-top boxes inserting it in a broadcaster TS. This way any software upgrade can be also accomplished without the patient involvement. A simple and

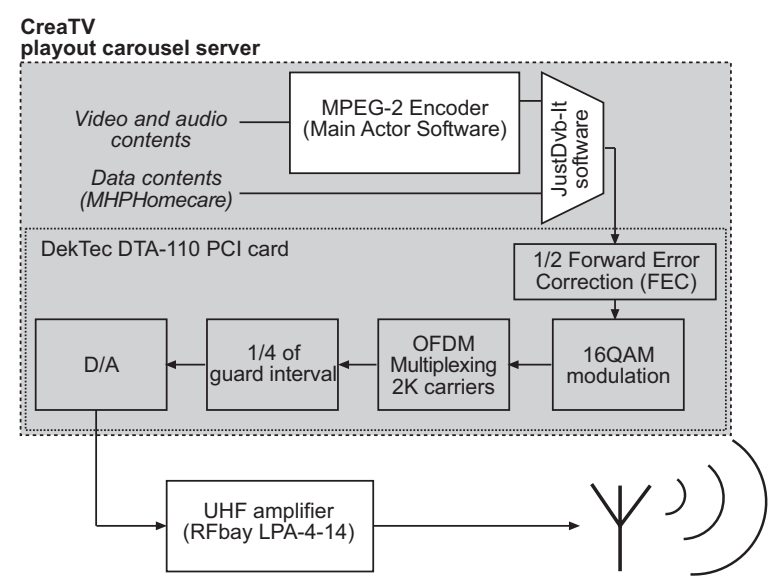

Fig. 4. The block diagram of our broadcast transmission system.

powerful smart-card identification and customization system has been proven to be effective in reducing the patient's interaction with the application. A proof-of-concept low-cost Base Station for the actual bio-signals acquisition and processing has been also presented. We are continuously improving its functionalities providing interfaces for commercial devices too (e.g. glucose meters).

\section{ACKNOWLEDGMENTS}

This work is part of the Personal e-Care project, which has been partially founded by INFN, the Nuclear Physics Italian Institute. The authors would like to thank CINECA [9] for its invaluable contribution to the setup of the broadcast transmission system.

\section{REFERENCES}

[1] D. Jung, K. Kim, G. Kim, D. Shim, M. Kim, B. Choi, and D. Suh, "Biosignal monitoring system for mobile telemedicine," in Proc. HEALTHCOM 2005 International Conference on Health Communication. IEEE, 2005.

[2] L. Pierucci and E. D. Re, "An interactive multimedia satellite telemedicine service," IEEE MultiMedia, vol. 07, no. 2, pp. 76-83.

[3] C. Mailhes, F. Castanié, S. Henrion, L. Lareng, A. Alonso, J.-L. Weber, B. Zeevi, P. Lochelongue, Y. Depeursinge, V. Kollias, and M. Ferhaoui, "The U-R-Safe telemedicine project: improving health care of the elderly," in Proc. of MIE2003, St.Malo, France, 2003.

[4] P. Loghelongue, "Satellite based telemedicine: Accessibility, quality and safety," in Proc. Tromso Telemedicine and eHealth Conference, Tromso, Norway, June, 2007.

[5] E. Kyriacou, S. Pavlopoulos, D. Koutsouris, A. Andreou, C. Pattichis, and C. Schizas, "Multipurpose health care telemedicine system," in Proc. of the 23rd Annual EMBS International Conference, Istanbul, Turkey, Oct. 2001.

[6] B. Calder, J. Courtney, B. Foote, L. Kyrnitszke, D. Rivas, C. Saito, J. V. Loo, and T. Ye, Java TV TM API Technical Overview: the JavaTV API Whitepaper, Nov. 2000, version 1.0.

[7] "Security and trust services API for J2ME (SATSA); JSR 177." [Online] Available: http://java.sun.com/products/satsa

[8] D. E. Gustafson, A. S. Willsky, S. K. Mitter, J.-Y.Wang, A. Akant, W. C. Kessel, and P. C. Doerschuk, "Automated VCG interpretation studies using signal analysis techniques," The Charles Stark Draper Laboratory Inc., Cambridge, MA 02139, Tech. Rep., jan 1977.

[9] "Cineca." [Online]. Available: http://www.cineca.tv 